\title{
TMAO-Activated Hepatocyte-Derived Exosomes Are Widely Distributed in Mice with Different Patterns and Promote Vascular Inflammation
}

\author{
Xiang Liu $\mathbb{D}^{1,},{ }^{1,2}$ Jiazichao Tu $\mathbb{D}^{1,2}$ Ziqin Zhou $\mathbb{D}^{1,2}$ Bingxin Huang ${ }^{10},{ }^{1,2}$ \\ Jianrong Zhou $\mathbb{D}^{1,2}$ and Jimei Chen $\mathbb{D}^{1,2}$ \\ ${ }^{1}$ Department of Cardiac Surgery, Guangdong Cardiovascular Institute, Guangdong Provincial People's Hospital, \\ Guangdong Academy of Medical Sciences, Guangzhou 510080, China \\ ${ }^{2}$ Guangdong Provincial Key Laboratory of South China Structural Heart Disease, Guangzhou 510080, China
}

Correspondence should be addressed to Jimei Chen; jimei_1965@outlook.com

Received 23 December 2021; Revised 14 January 2022; Accepted 15 January 2022; Published 14 February 2022

Academic Editor: Jianqin Wei

Copyright (c) 2022 Xiang Liu et al. This is an open access article distributed under the Creative Commons Attribution License, which permits unrestricted use, distribution, and reproduction in any medium, provided the original work is properly cited.

Background. Trimethylamine-N-oxide (TMAO) has been shown to be an important player in cardiovascular disease (CVD) by promoting vascular inflammation and endothelial dysfunction. We recently found that exosomes (Exos) released from TMAOactivated hepatocytes (TMAO-Exos) could significantly induce inflammation and endothelial dysfunction. However, understandings of how are the Exos secreted by hepatocytes, where are they distributed in vivo, and what effects will they have on vascular inflammation remain limited. The present study aimed to explore the hub genes involved in the production of TMAOExos and their distributions in vivo and effects on inflammation. Methods. The transcriptome profiles of primary rat hepatocytes stimulated with TMAO were obtained from the GSE135856 dataset in the Gene Expression Omnibus repository, and the hub genes associated with Exos were screened and verified by qPCR. Next, Exos derived from TMAO-treated hepatocytes were isolated using differential centrifugation and given intravenously to mice. After $24 \mathrm{~h}$, the distributions of DiI-labelled Exos were visualized with a fluorescence microscope, and the levels of proinflammatory genes in the aorta were detected by qPCR. Results. Phgdh, Mdh2, Echs1, Rap2a, Gpd1l, and Slc3a2 were identified as hub genes that may be involved in the production of TMAO-Exos. And TMAO-Exos were found to be efficiently taken up by cardiomyocytes, hepatocytes, and endothelial cells in the aorta and gastrocnemius muscle. Furthermore, TMAO-Exos, but not control-Exos, could significantly promote the mRNA expressions of Tnf, Icam 1, Sele, and Cox-2 in the aorta. Conclusions. We provided clues about how TMAO may stimulate hepatocytes to produce Exos and further offered evidence that Exos secreted by TMAO-treated hepatocytes could be widely distributed in vivo and promote vascular inflammation.

\section{Introduction}

There is increasing evidence that gut microbiota and its metabolites play a key role in the pathogenesis and development of cardiovascular disease (CVD) [1]. Thereinto, trimethylamine-N-oxide (TMAO) has been found to be an independent risk factor for adverse cardiovascular events [2-6], which may be related to excessive vascular inflammation and endothelial dysfunction provoked [7-10]. It has been shown that dietary precursors such as choline, betaine, and L-carnitine can be metabolized into trimethylamine in the gut flora and further catalyzed into trimethylamine-Noxide (TMAO) in the liver $[2,5,11]$. However, the mechanisms underlying TMAO are still not completely understood.

Exosomes (Exos) have gained a growing concern for the pivotal roles in cardiovascular physiology [12]. Exos are nanosized membrane vesicles produced by nearly all types of cells, ranging from approximately 30 to $100 \mathrm{~nm}$ in diameter and containing a variety of bioactive molecules [12-14]. Recent research has found that Exos derived from hepatocytes play an important role in inflammation, endothelial 
function, and metabolic disorders [15-19]. In the latest work, we found that Exos secreted by TMAO-treated hepatocytes (TMAO-Exos) contained a distinctive profile of miRNAs compared to those from the TMAO-free group (control-Exos), and furthermore, TMAO-Exos could notably promote inflammation, damage vascular endothelial cells (VECs), and impair endothelium-dependent vasodilation [20]. However, understandings of how these Exos are produced by hepatocytes, where are they distributed in vivo, and what effects will they have on vascular inflammation remain limited.

In the present study, we first obtained the transcriptome profiles of primary rat hepatocytes stimulated with or without TMAO [21] and then identified the hub genes related to Exos. Next, Exos were isolated from hepatocytes treated with or without TMAO and given intravenously to mice, and then their distributions in the heart, liver, aorta, and gastrocnemius muscle and effects on vascular inflammation were examined.

\section{Materials and Methods}

2.1. Hepatic Gene Expressions Induced by TMAO. The transcriptome profiles of primary rat hepatocytes stimulated with or without TMAO $(50 \mu \mathrm{mol} / \mathrm{L})$ for $30 \mathrm{~h}$ were obtained from the GSE135856 dataset in the Gene Expression Omnibus repository $[21,22]$. The microarray experiments were performed using a single Affymetrix Clariom S Rat array (Affymetrix, Santa Clara, CA, USA) with three biological repetitions. The open-source Bioconductor packages, affy and limma, were used to process the data [21].

2.2. Enrichment Analysis. The differentially expressed genes $(P<0.05)$ related to Exos were screened and visualized on a heatmap constructed in R. DAVID (Database for Annotation, Visualization, and Integrated Discovery) was used for investigating the functional annotation of genes. GO (gene ontology) analysis was performed to elaborate the biological process, STRING (Search Tool for the Retrieval of Interacting Genes/Proteins) database (v11.0) [23] was used for analyzing the protein-protein interaction (PPI), and networks were constructed on Cytoscape platform (v3.8.2) [24]. CytoHubba plugin was used to identify hub genes with a threshold value $>0.4$, and the color of the nodes represented the degree of gene interaction.

2.3. Cell Culture and Exos Isolation. The procedures were performed as described in our recent study [20]. In brief, AML12 cells (iCell Bioscience Inc., Shanghai) were cultured in DMEM/F12 (iCell Bioscience Inc., Shanghai) containing Exos-depleted serum (ViVaCell, Shanghai) and treated with TMAO (Tokyo Chemical Industry Co., Ltd.) at a physiological concentration of $50 \mu \mathrm{mol} / \mathrm{L}$ for $48 \mathrm{~h}$ (TMAO-Exos). The untreated group served as the control (control-Exos). Exos were isolated and purified from the culture supernatant using differential centrifugation and then resuspended in PBS.
2.4. Exos Identification and Labelling. The protein levels of the Exos were measured using the BCA protein assay kit (Thermo Fisher Scientific, MA, USA). The ultrastructure of the Exos was inspected using a transmission electron microscope (JEM 1200-EX, Japan). In brief, Exos suspensions were loaded on 200-mesh formvar-coated grids and then negatively stained with phosphotungstic acid. The samples were observed under a transmission electron microscope at a voltage of $100 \mathrm{kV}$. The concentration and size distribution of the Exos were detected by nanoparticle tracking analysis (Nanosight NS300, Malvern, UK). Exosomal markers of CD9 and TSG101 and the negative marker of calnexin were detected by western blotting. In brief, the samples were separated by SDS-PAGE and transferred onto Millipore polyvinylidene difluoride membranes. The membranes were incubated overnight at $4^{\circ} \mathrm{C}$ with the primary antibodies of CD9 (Zenbio, Chengdu, China), TSG101 (Zenbio, Chengdu, China), and calnexin (Affinity Biosciences, Jiangsu, China) and visualized with enhanced chemiluminescence reagent (Millipore, MA, USA). Exos were labelled with DiI (Beyotime Biotechnology, Shanghai, China) for in vivo tracer experiments.

2.5. Animal Experiments. All experiments conform to the protocols approved by the Institutional Animal Care and Use Committee of Guangdong Provincial People's Hospital. Male wild-type C57BL/6 mice (six weeks old) were purchased from the Experimental Animal Center of Sun Yat-sen University. The mice were intravenously injected with $30 \mu \mathrm{g}$ of Exos resuspended in $100 \mu \mathrm{L}$ of PBS. After $24 \mathrm{~h}$, the mice were anesthetized by pentobarbital sodium $(50 \mathrm{mg} / \mathrm{kg}$ ), and then the hearts, thoracic aortas, livers, and gastrocnemius muscles were collected for subsequent study.

2.6. Fluorescence Detection. The hearts, thoracic aortas, livers, and gastrocnemius muscles were fixed in $4 \%$ paraformaldehyde and then dehydrated and embedded in paraffin. Samples underwent dewaxing and antigen retrieval. The slides were blocked in $10 \%$ goat serum for $30 \mathrm{~min}$ at room temperature and then incubated with the primary antibody of CD31 (Abcam, MA, USA) overnight. Slides were incubated with Alexa Fluor ${ }^{\circledR} 488$ donkey anti-rabbit IgG $(\mathrm{H}+\mathrm{L})$. Wheat germ agglutinin (WGA, Sigma-Aldrich, USA) staining was used to outline the cardiomyocytes. Slides were then washed and stained with DAPI (Solarbio, Beijing, China). The positive signals were detected with a fluorescence microscope (Olympus, Tokyo, Japan).

\subsection{Quantitative Polymerase Chain Reaction.} Quantitative polymerase chain reaction (qPCR) was performed as described in our previous study [25]. In brief, total RNA was extracted using TRIzol reagent (Invitrogen, USA), and concentration was measured using the NanoDrop 2000 spectrophotometer (Thermo Fisher Scientific, MA, USA). Then, RNA was reversely transcribed into cDNA using Color Reverse Transcription Kit (EZBioscience, CA, USA), 
TABLE 1: qPCR primers for mRNA (Mus musculus) used in the study.

\begin{tabular}{lcc}
\hline Name & Forward sequence & Reverse sequence \\
\hline Phgdh & CCAGGTGGTTACACAAGGAACA & TTCACGTCTGCCTGCTTAGATG \\
Mdh2 & TTTGTGGCAGAGCTAAAGGGTT & GTACACTGAGAGATCAGGGGGA \\
Echs1 & AATGGAGATGGTCCTCACTGGT & TCTGCACATTGGATGGCTTCTT \\
Rap2a & CAGCAGAGCTTCCAAGACATCA & CTCTCCAGGTCCACTTTGTTCC \\
Gpd11 & TCCGACATCATCCGAGAGAAGA & AAGGCCGTTCTGCATCACTTT \\
Slc3a2 & GGCCCAATTCACAAGAACCAGA & TGGGAGTGAGGTCCAAAATGATG \\
Hyou1 & GATCTTCGGGTATTTGGCTCCC & TAAAGTGGGCCTTGATGCCTTT \\
Hspa9 & AACTCCTGTGTGGTGTTATGG & CAAGTCGTTCTCCATCTGCTGT \\
Psat1 & GGGTGGAGTTTGACTTCGTACC 2 & TCTTCTGAGCACCAGCGAAAAT \\
Tnf & GAGGCTGAGAACACTGCCATAC & TGCGAAGGAGTTACAATCACCG \\
Icam 1 & CTGTAGCCCACGTCGTAGC & TTGAGATCCATGCCGTTG \\
Sele & CCCACGCTACCTCTGCTC & GATGGATACCTGAGCATCACC \\
Cox-2 & ATGCCTCGCGCTTTCTCTC & GTAGTCCCGCTGACAGTATGC \\
Gapdh & TTCAACACACTCTATCACTGGC & AGAAGCGTTTGCGGTACTCAT \\
\hline
\end{tabular}

Phgdh: 3-phosphoglycerate dehydrogenase; Mdh2: malate dehydrogenase 2, NAD (mitochondrial); Echs1: enoyl coenzyme A hydratase, short chain, 1, mitochondrial; Rap2a: Ras-related protein 2a; Gpd1l: glycerol-3-phosphate dehydrogenase 1-like; Slc3a2: solute carrier family 3 (activators of dibasic and neutral amino acid transport), member 2; Hyou1: hypoxia upregulated 1; Hspa9: heat shock protein 9; Psat1: phosphoserine aminotransferase 1; Pck2: phosphoenolpyruvate carboxykinase 2 (mitochondrial); Tnf: tumor necrosis factor; Icam1: intercellular adhesion molecule-1; Sele: selectin, endothelial cell; Cox-2: cyclooxygenase-2; Gapdh glyceraldehyde-3-phosphate dehydrogenase.

and qPCR was performed on Bio-Rad CFX-96 (Bio-Rad, CA, USA) with Color SYBR Green qPCR Master Mix (EZBioscience, CA, USA). The expressions were normalized to Gapdh. The qPCR primers used in the study are listed in Table 1.

2.8. Statistical Analysis. Statistical analysis was conducted using SPSS 20.0 software (SPSS Inc., Chicago, IL, USA), and the graphs were plotted by GraphPad Prism (version 9.3.0, San Diego, USA). Data were presented as mean \pm standard error of the mean (SEM). For continuous variables with normal distribution, the comparisons between two groups were performed with independent $t$-test. For continuous variables with nonnormal distribution, the comparisons between two groups were performed with Wilcoxon ranksum test. A $P$ value $<0.05$ was considered statistically significant.

\section{Results}

3.1. Exos-Related Genes in Hepatocytes Were Dysregulated by TMAO. Microarray assay was performed to determine the expression profiles of genes in primary rat hepatocytes stimulated with TMAO. Compared to the untreated group, a total of 101 genes related to Exos changed significantly $(P<0.05)$, and among these genes, 43 were upregulated and 58 were downregulated (Figure 1(a)). GO analysis was used to explore the predominant biological processes, and the results showed that the differentially expressed genes (DEGs) were significantly enriched in secretion, peptide metabolic process, export from the cell, membrane fusion, and small-molecule biosynthetic process (Figure 1(b)). Besides, the PPI networks were constructed to further elaborate the interactions among the DEGs and identified Phgdh, Mdh2, Echs1, Rap2a, Gpd1l, Slc3a2, Hyou1, Hspa9, Psat1, and Pck2 as the top 10 hub genes (Figure 1(c)).
3.2. Verification of the Hub Genes. To verify the changes of the hub genes, AML12 cells were stimulated with or without TMAO for $48 \mathrm{~h}$, and the mRNA levels of the top $10 \mathrm{hub}$ genes were detected by qPCR. It was shown that TMAO remarkably enhanced the mRNA expressions of Phgdh, Mdh2, Echs1, Rap2a, and Gpd1l (Figures 2(a)-2(e)) and reduced Slc3a2 levels (Figure 2(f)). However, Hyou1, Hspa9, $P s a t 1$, and Pck2 remained unchanged (Figures 2(g)-2(j)).

3.3. Isolation and Identification of Exos. Nanovesicles with a cup-shaped morphology and a typical size around $100 \mathrm{~nm}$ were isolated and purified from the cell culture supernatant (Figure 3(a)). The size distribution profiles and concentrations of the Exos showed no significant differences between the two groups (Figure 3(b)). Exosomal markers of CD9 and TSG101 were mainly enriched in control-Exos and TMAOExos, and the negative marker of calnexin was detected only in the whole cell lysate (Figure 3(c)).

\subsection{Exos Were Widely Distributed In Vivo with Different} Patterns. TMAO-Exos were labelled with DiI and administered intravenously. The results clearly showed that Exos could be efficiently taken up by cardiomyocytes (Figure 4(a)), hepatocytes (Figure 4(b)), and endothelial cells in the aorta (Figure 4(c)). It was worth noting that these Exos were predominantly localized in the nuclei of hepatocytes. And unlike in cardiomyocytes, these Exos appeared to keep themselves out of the skeletal muscle cells and preferentially located in the endothelial cells, as indicated by the same subcellular localizations of Exos and CD31 proteins in the gastrocnemius muscle (Figure 4(d)).

3.5. TMAO-Exos Promoted Vascular Inflammation. The in vivo tracer experiments clearly showed that TMAO-Exos could target endothelial cells in the aorta and gastrocnemius 


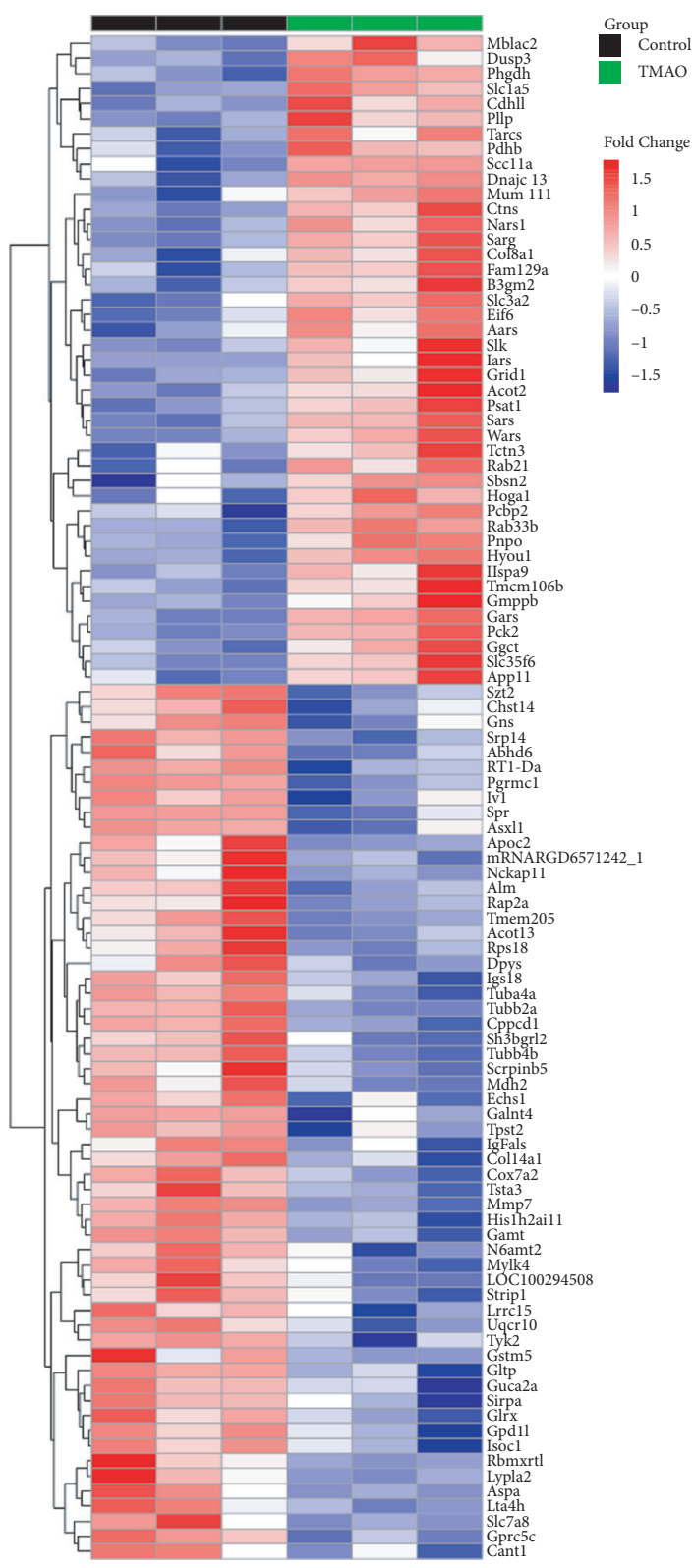

(a)

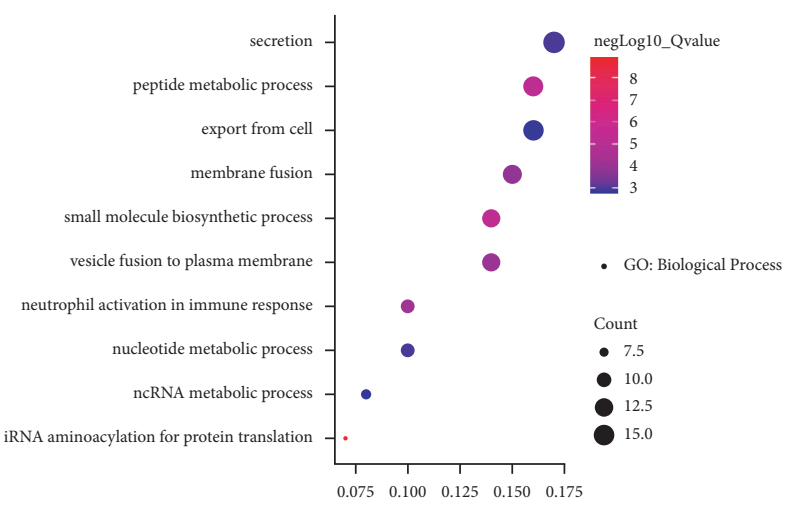

Figure 1: Continued. 


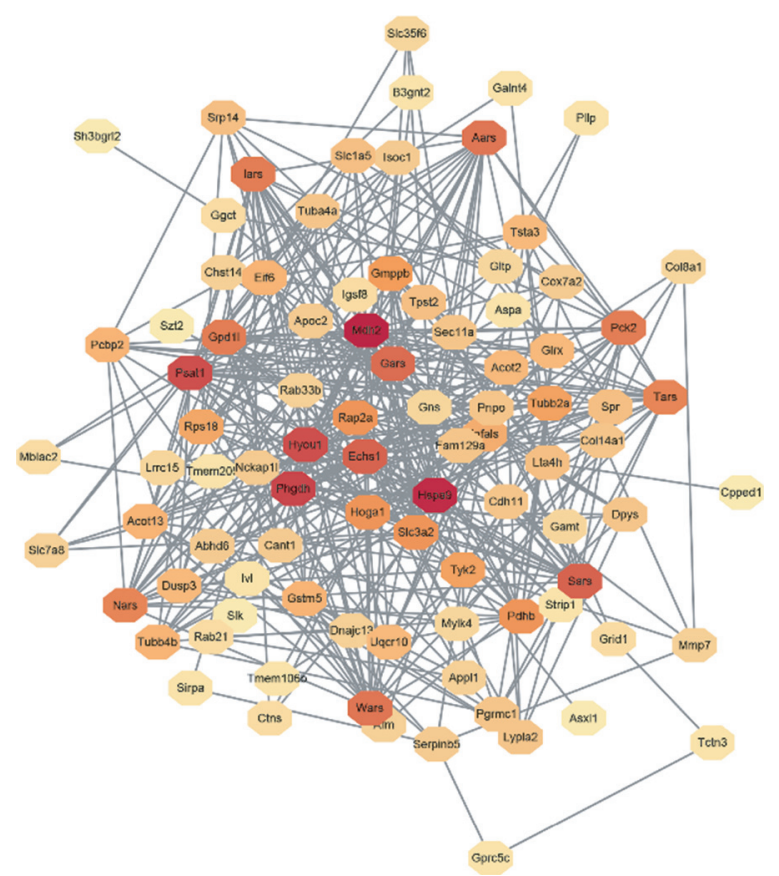

(c)

FIgURE 1: Exos-related genes in hepatocytes were dysregulated by TMAO. (a) A total of 101 genes related to Exos changed significantly $(P<0.05)$ after TMAO treatment. (b) The differentially expressed genes were significantly enriched in the biological processes of secretion, peptide metabolic process, export from the cell, membrane fusion, and small-molecule biosynthetic process. (c) Phgdh, Mdh2, Echs1, Rap2a, Gpd1l, Slc3a2, Hyou1, Hspa9, Psat1, and Pck2 were identified as the top 10 hub genes.

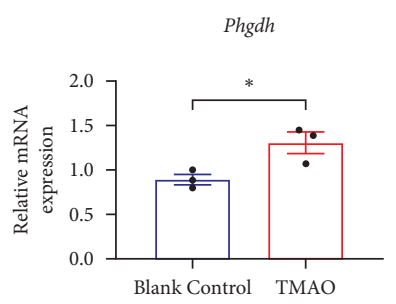

(a)

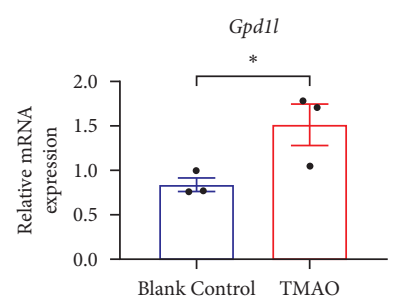

(e)

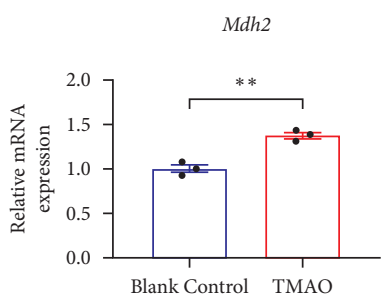

(b)

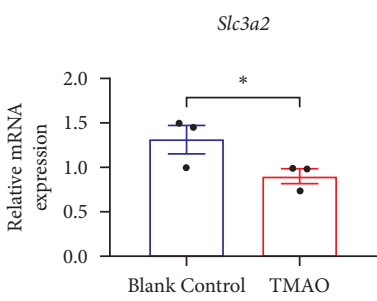

(f)

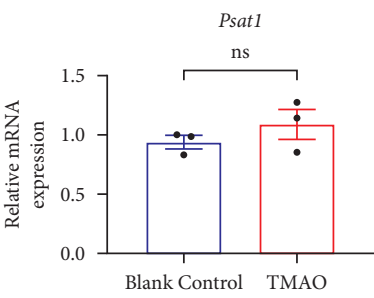

(i)

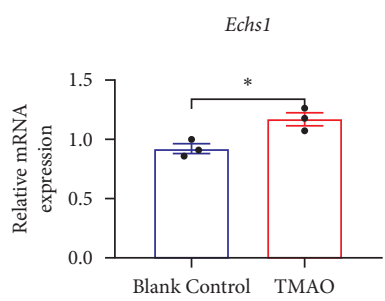

(c)

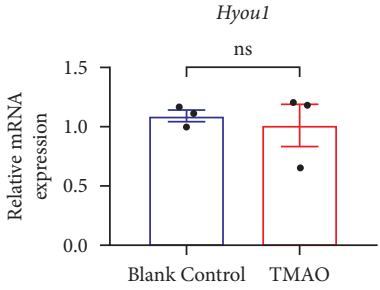

(g)

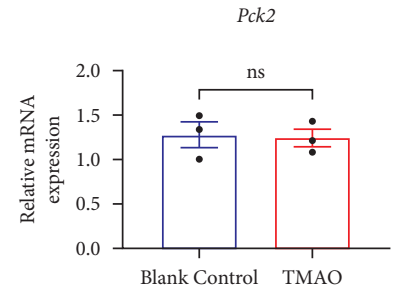

(j)

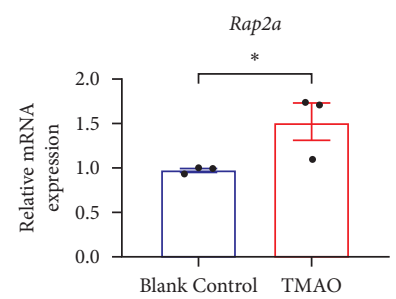

(d)

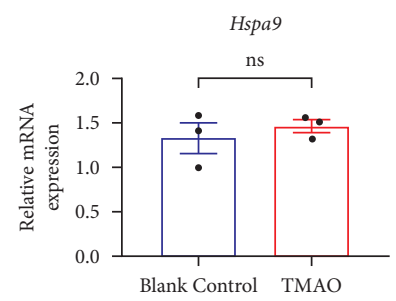

(h)

Figure 2: Verification of the top 10 hub genes. (a-e) TMAO remarkably enhanced the mRNA expressions of Phgdh, Mdh2, Echs1, Rap2a, and Gpd1l and (f) reduced Slc3a2 levels. (g-j) Hyou1, Hspa9, Psat1, and Pck2 remained unchanged. qPCR was used to determine the mRNA expressions $(n=3) .{ }^{*} P<0.05$ vs. the blank control group. ns indicates not significant. 


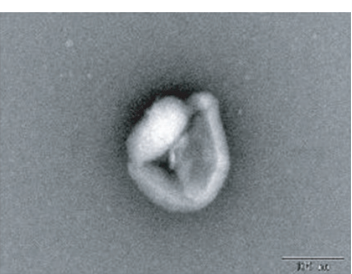

control-Exos

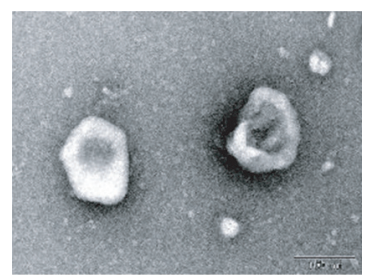

TMAO-Exos
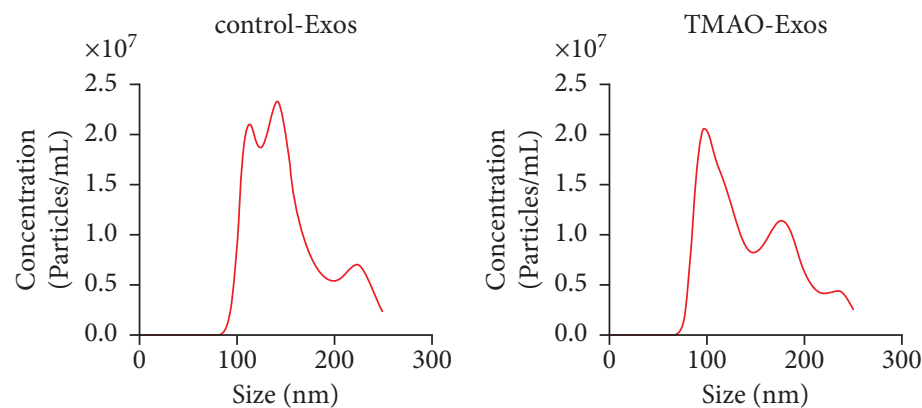

(b)

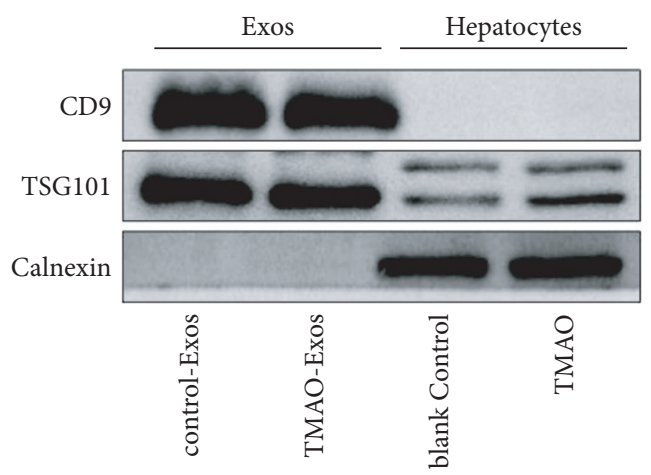

(c)

Figure 3: Isolation and identification of Exos. (a) Nanovesicles with a cup-shaped morphology and a typical size around $100 \mathrm{~nm}$ were isolated and purified from the cell culture supernatant. Bar: $100 \mathrm{~nm}$. (b) The size distribution of the Exos showed no significant difference between control-Exos and TMAO-Exos. (c) Exosomal markers of CD9 and TSG101 were enriched in Exos groups, and the negative marker of calnexin was detected only in the whole cell lysate.

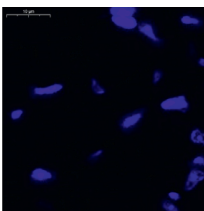

DAPI

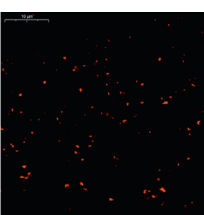

Dil-Exos

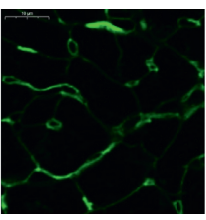

WGA

(a)

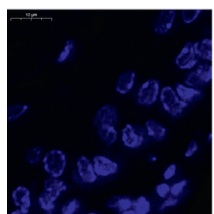

DAPI

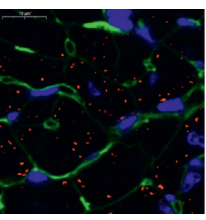

Merged

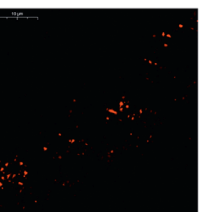

Dil-Exos

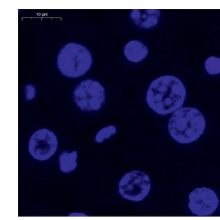

DAPI

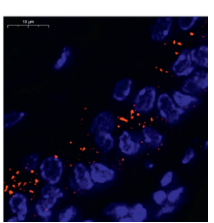

Merged

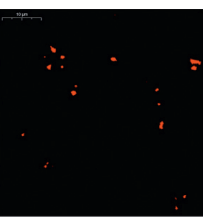

Dil-Exos

(b)

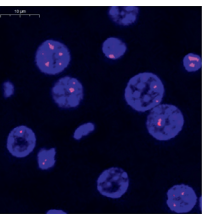

Merged

(c)

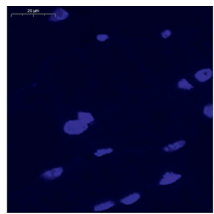

DAPI

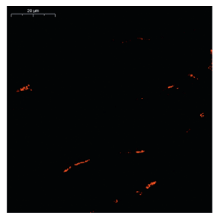

Dil-Exos

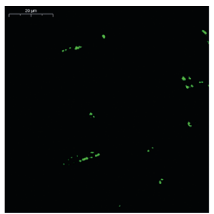

CD31

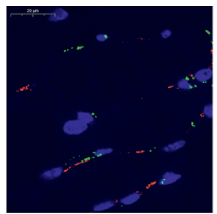

Merged

(d)

Figure 4: Exos were widely distributed in vivo with different patterns. Exos secreted by TMAO-treated hepatocytes could be efficiently captured by (a) cardiomyocytes, (b) hepatocytes, and (c) endothelial cells in the aorta. (d) The Exos appeared to keep themselves out of the skeletal muscle cells and preferentially located in the endothelial cells. 


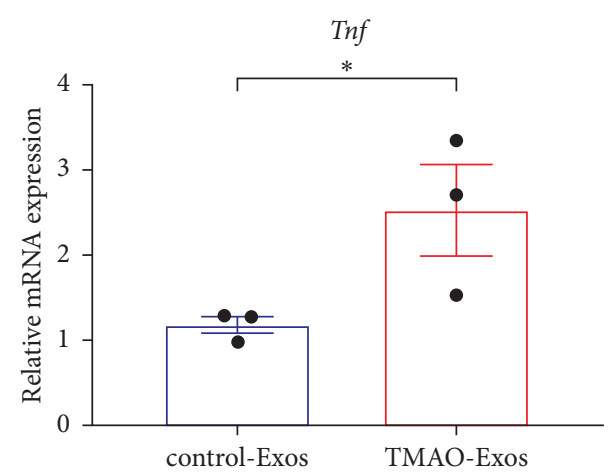

(a)

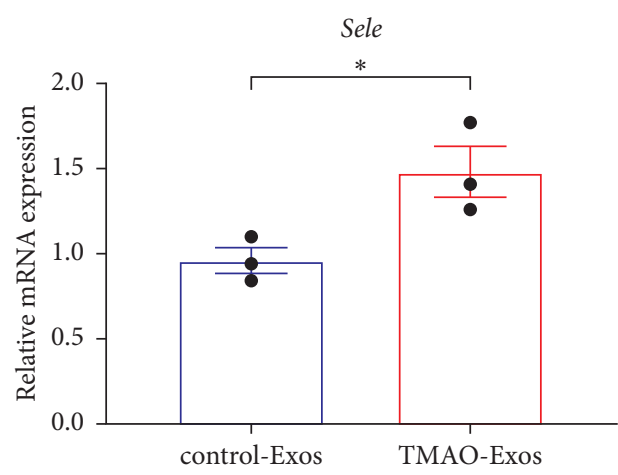

(c)

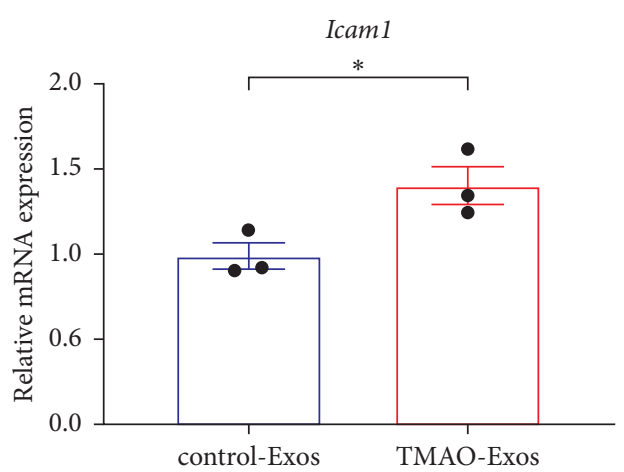

(b)

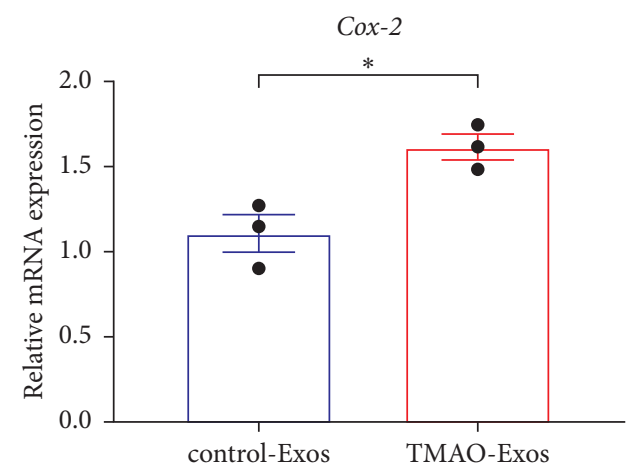

(d)

FIgURE 5: TMAO-Exos promoted the mRNA expressions of inflammatory genes in aortas. TMAO-Exos notably enhanced the mRNA levels of (a) Tnf, (b) Icam1, (c) Sele, and (d) Cox-2. qPCR was used to determine the mRNA expressions $(n=3) .{ }^{*} P<0.05$ vs. the control-Exos group.

muscle, so we assessed the effects of TMAO-Exos on the mRNA expressions of inflammatory genes in aortas. Compared to control-Exos, TMAO-Exos significantly promoted the mRNA expressions of Tnf, Icam 1, Sele, and Cox-2 (Figures 5(a)-5(d)).

\section{Discussion}

In the current work, we first identified the hub genes in hepatocytes after TMAO treatment, which may be related to the production of TMAO-Exos. Next, we provided evidence that TMAO-Exos could be taken up by several tissues including the heart, liver, aorta, and gastrocnemius muscle with different distribution patterns. And finally, we found that TMAO-Exos, but not control-Exos, could significantly promote the mRNA expressions of $\operatorname{Tnf}$, Icam 1 , Sele, and Cox -2 in the aorta.

There is a close relationship between TMAO and the liver. TMAO, at a physiological concentration of $50 \mu \mathrm{mol} / \mathrm{L}$, has been shown to be able to target hepatocytes and result in the abnormal expressions of hepatic genes, thus exerting an influence on metabolic disorder [21]. Moreover, we recently found that TMAO $(50 \mu \mathrm{mol} / \mathrm{L})$ could stimulate hepatocytes to release functional Exos [20]. However, the molecular pathways and hub genes behind it remain unknown. It was found that the fusion of multivesicular bodies (MVBs) with the plasma membrane is critical for the extracellular secretion of Exos, and intracellular molecules and pathways such as Rab27a/b and V-ATPase-mediated acidification of MVBs are implicated [26]. An interference study showed that HGS, Alix, TSG101, and nSmase2 were necessary for the Exos production by normal or ethanol-treated hepatocytes [27]. And other studies revealed that Tsg101 and Vps4a played a pivotal role in exosomal secretion $[28,29]$. Based on the aforementioned study [21], a Exos-related gene set was selected to identify the predominant biological processes and hub genes which responded to TMAO treatment. The results showed that the DEGs were mainly enriched in secretion, peptide metabolic process, export from the cell, membrane fusion, and small-molecule biosynthetic process, which suggested that these DEGs may be related to the synthesis and secretion of TMAO-Exos. Furthermore, we verified that Phgdh, Mdh2, Echs1, Rap2a, and Gpd1l were upregulated, and Slc3a2 was downregulated by TMAO. Phgdh and Mdh2 are classified into the biological process of "small-molecule biosynthetic process." The protein encoded by Phgdh is essential for serine synthesis and contributes to glucose and lipid homeostasis [30-32]. And gene Mdh2 encodes a mitochondrial enzyme that plays a pivotal role in the malateaspartate shuttle and glucose homeostasis [33, 34]. In addition, $S l c 3 a 2$ encodes a membrane protein and is involved in amino acid transport and endoplasmic reticulum stress [35-37]. However, the results simply provided some early clues about how TMAO may stimulate hepatocytes to 
produce Exos, and the exact mechanisms remain to be further elucidated by using the gain- and loss-of-function strategies.

Similarly, the distribution patterns of Exos remain poorly defined. It was suggested that the processes may depend on the distinctive factors or receptors located on the recipient cell surfaces [38]. However, Horibe et al. found that Exos could be nonselectively incorporated into recipient cells via a different mechanism, which may depend on the recipient cells rather than the donor cells [39]. In the present work, we showed that TMAO-Exos could be distributed in tissues including the heart, liver, aorta, and gastrocnemius muscle after systemic administration, suggesting that these Exos spread widely in vivo through the bloodstream. However, it seemed that the distribution of these Exos varied depending on the recipient cell type, as indicated by the observations that they could be incorporated by cardiomyocytes, but appeared to keep themselves out of the skeletal muscle cells and preferentially located in the endothelial cells. In addition, it was interesting to see that these Exos were able to be captured by hepatocytes and localized in the nuclei. These findings were consistent with previous research, in which Exos were able to be captured by the donor cells themselves [27, 39]. Furthermore, we found that TMAO-Exos could significantly promote the mRNA expressions of Tnf, Icam 1, Sele, and Cox-2 in the aortas. These proinflammatory genes have been shown to play pivotal roles in the regulation of inflammation, endothelial dysfunction, and atherosclerosis [40-43].

\section{Conclusions}

In the current study, we provided clues about how TMAO may stimulate hepatocytes to produce Exos and further offered evidence that Exos secreted by TMAO-treated hepatocytes could be widely distributed in vivo and promote vascular inflammation. These findings may help to advance our understanding of the mechanisms by which TMAO promotes CVD.

\section{Data Availability}

The data relevant to this study are available from the corresponding author upon reasonable request.

\section{Conflicts of Interest}

The authors declare that they have no conflicts of interest regarding the publication of this paper.

\section{Authors' Contributions}

Jimei Chen contributed to the concept, designed the study, directed the research, and revised the manuscript. Xiang Liu, Jiazichao Tu, Ziqin Zhou, Bingxin Huang, and Jianrong Zhou performed the experiments and analyzed the data. Xiang Liu drafted the manuscript. All authors read and approved the final manuscript.

\section{Acknowledgments}

This work was supported by the Guangdong Peak Project (no. DFJH2019), Science and Technology Projects in Guangzhou (no. 202102021149), and Postdoctoral Scientific Research Start-up Fund Project of Guangdong Provincial People's Hospital (no. BY012021052).

\section{References}

[1] W. H. W. Tang, T. Kitai, and S. L. Hazen, "Gut microbiota in cardiovascular health and disease," Circulation Research, vol. 120, no. 7, pp. 1183-1196, 2017.

[2] Z. Wang, E. Klipfell, B. J. Bennett et al., "Gut flora metabolism of phosphatidylcholine promotes cardiovascular disease," Nature, vol. 472, no. 7341, pp. 57-63, 2011.

[3] W. Zhu, J. C. Gregory, E. Org et al., "Gut microbial metabolite tmao enhances platelet hyperreactivity and thrombosis risk," Cell, vol. 165, no. 1, pp. 111-124, 2016.

[4] X. S. Li, S. Obeid, R. Klingenberg et al., "Gut microbiotadependent trimethylamine n-oxide in acute coronary syndromes: a prognostic marker for incident cardiovascular events beyond traditional risk factors," European Heart Journal, vol. 38, no. 11, pp. 814-824, 2017.

[5] W. H. W. Tang, Z. Wang, B. S. Levison et al., "Intestinal microbial metabolism of phosphatidylcholine and cardiovascular risk," New England Journal of Medicine, vol. 368, no. 17, pp. 1575-1584, 2013.

[6] W. H. W. Tang, Z. Wang, Y. Fan et al., "Prognostic value of elevated levels of intestinal microbe-generated metabolite trimethylamine-N-oxide in patients with heart failure," Journal of the American College of Cardiology, vol. 64, no. 18, pp. 1908-1914, 2014.

[7] G. Yang, C.-C. Lin, Y. Yang et al., "Nobiletin prevents trimethylamine oxide-induced vascular inflammation via inhibition of the NF- $\kappa$ B/MAPK pathways," Journal of Agricultural and Food Chemistry, vol. 67, no. 22, pp. 61696176, 2019.

[8] X. Sun, X. Jiao, Y. Ma et al., “Trimethylamine n-oxide induces inflammation and endothelial dysfunction in human umbilical vein endothelial cells via activating ros-txnip-nlrp3 inflammasome," Biochemical and Biophysical Research Communications, vol. 481, no. 1-2, pp. 63-70, 2016.

[9] M. M. Seldin, Y. Meng, and H. Qi, "Trimethylamine n-oxide promotes vascular inflammation through signaling of mitogen-activated protein kinase and nuclear factor-kappab," Journal of American Heart Association, vol. 5, no. 2, 2016.

[10] X. Zhang, Y. Li, P. Yang et al., "Trimethylamine-N-Oxide promotes vascular calcification through activation of NLRP3 (Nucleotide-Binding domain, leucine-rich-containing family, pyrin domain-containing-3) inflammasome and NF- $\kappa \mathrm{B}$ (nuclear factor $\kappa \mathrm{B}$ ) signals," Arteriosclerosis, Thrombosis, and Vascular Biology, vol. 40, no. 3, pp. 751-765, 2020.

[11] R. A. Koeth, Z. Wang, B. S. Levison et al., "Intestinal microbiota metabolism of l-carnitine, a nutrient in red meat, promotes atherosclerosis," Nature Medicine, vol. 19, no. 5, pp. 576-585, 2013.

[12] A. Ibrahim and E. Marbán, "Exosomes: fundamental biology and roles in cardiovascular physiology," Annual Review of Physiology, vol. 78, no. 1, pp. 67-83, 2016.

[13] M. Vidal, "Exosomes: revisiting their role as "garbage bags", Traffic, vol. 20, no. 11, pp. 815-828, 2019. 
[14] S. Kourembanas, "Exosomes: vehicles of intercellular signaling, biomarkers, and vectors of cell therapy," Annual Review of Physiology, vol. 77, no. 1, pp. 13-27, 2015.

[15] F. Momen-Heravi, S. Bala, K. Kodys, and G. Szabo, "Exosomes derived from alcohol-treated hepatocytes horizontally transfer liver specific mirna-122 and sensitize monocytes to lps," Scientific Reports, vol. 5, no. 1, p. 9991, 2015.

[16] Y. Ji, Z. Luo, H. Gao et al., "Hepatocyte-derived exosomes from early onset obese mice promote insulin sensitivity through mir-3075," Nature Metabolism, vol. 3, no. 9, pp. 1163-1174, 2021.

[17] F. Jiang, Q. Chen, W. Wang, Y. Ling, Y. Yan, and P. Xia, "Hepatocyte-derived extracellular vesicles promote endothelial inflammation and atherogenesis via microrna-1," Journal of Hepatology, vol. 72, no. 1, pp. 156-166, 2020.

[18] P. Hirsova, S. H. Ibrahim, A. Krishnan et al., "Lipid-induced signaling causes release of inflammatory extracellular vesicles from hepatocytes," Gastroenterology, vol. 150, no. 4, pp. 956-967, 2016.

[19] X. Luo, S.-Z. Luo, Z.-X. Xu et al., "Lipotoxic hepatocytederived exosomal mir-1297 promotes hepatic stellate cell activation through the pten signaling pathway in metabolicassociated fatty liver disease," World Journal of Gastroenterology, vol. 27, no. 14, pp. 1419-1434, 2021.

[20] X. Liu, Y. Shao, J. Tu et al., "Trimethylamine-n-oxide-stimulated hepatocyte-derived exosomes promote inflammation and endothelial dysfunction through nuclear factor-kappa $b$ signaling," Annals of Translational Medicine, vol. 9, no. 22, p. 1670, 2021.

[21] S. Chen, A. Henderson, M. C. Petriello et al., "Trimethylamine n-oxide binds and activates perk to promote metabolic dysfunction," Cell Metabolism, vol. 30, no. 6, pp. 1141-1151, 2019.

[22] T. Barrett, S. E. Wilhite, P. Ledoux et al., "Ncbi geo: archive for functional genomics data sets--update," Nucleic Acids Research, vol. 41, no. Database issue, pp. D991-D995, 2013.

[23] D. Szklarczyk, A. L. Gable, D. Lyon et al., "String v11: proteinprotein association networks with increased coverage, supporting functional discovery in genome-wide experimental datasets," Nucleic Acids Research, vol. 47, no. D1, pp. D607-D613, 2019.

[24] P. Shannon, A. Markiel, O. Ozier et al., "Cytoscape: a software environment for integrated models of biomolecular interaction networks," Genome Research, vol. 13, no. 11, pp. 2498-2504, 2003.

[25] L. Zhou, X. Liu, Z.-Q. Wang et al., "Simvastatin treatment protects myocardium in noncoronary artery cardiac surgery by inhibiting apoptosis through mir-15a-5p targeting," Journal of Cardiovascular Pharmacology, vol. 72, no. 4, pp. 176-185, 2018.

[26] M. Mathieu, L. Martin-Jaular, G. Lavieu, and C. Théry, "Specificities of secretion and uptake of exosomes and other extracellular vesicles for cell-to-cell communication," Nature Cell Biology, vol. 21, no. 1, pp. 9-17, 2019.

[27] L. Chen, R. Chen, S. Kemper, and D. R. Brigstock, "Pathways of production and delivery of hepatocyte exosomes," Journal of Cell Communication and Signaling, vol. 12, no. 1, pp. 343-357, 2018.

[28] S. Bänfer, D. Schneider, J. Dewes et al., "Molecular mechanism to recruit galectin-3 into multivesicular bodies for polarized exosomal secretion," Proceedings of the National Academy of Sciences, vol. 115, no. 19, pp. E4396-E4405, 2018.

[29] Q. Han, L. Lv, J. Wei et al., "Vps4A mediates the localization and exosome release of $\beta$-catenin to inhibit epithelial- mesenchymal transition in hepatocellular carcinoma," Cancer Letters, vol. 457, pp. 47-59, 2019.

[30] W.-C. Sim, W. Lee, H. Sim et al., "Downregulation of phgdh expression and hepatic serine level contribute to the development of fatty liver disease," Metabolism, vol. 102, Article ID 154000, 2020.

[31] M. Hamano, K. Esaki, and K. Moriyasu, "Hepatocyte-specific phgdh-deficient mice culminate in mild obesity, insulin resistance, and enhanced vulnerability to protein starvation," Nutrients, vol. 13, no. 10, 2021.

[32] Y. P. Kang, A. Falzone, M. Liu et al., "Phgdh supports liver ceramide synthesis and sustains lipid homeostasis," Cancer \& Metabolism, vol. 8, no. 1, p. 6, 2020.

[33] T. Wang, W. Yao, J. Li, Q. He, Y. Shao, and F. Huang, “Acetylcoa from inflammation-induced fatty acids oxidation promotes hepatic malate-aspartate shuttle activity and glycolysis," American Journal of Physiology - Endocrinology And Metabolism, vol. 315, no. 4, pp. E496-E510, 2018.

[34] P. Jungtrakoon Thamtarana, A. Marucci, and L. Pannone, "Gain of function of malate dehydrogenase 2 (mdh2) and familial hyperglycemia," The Journal of Cinical Endocrinology and Metabolism, 2021.

[35] N. C. Henderson, E. A. Collis, A. C. Mackinnon et al., "CD98hc (SLC3A2) interaction with $\beta 1$ integrins is required for transformation," Journal of Biological Chemistry, vol. 279, no. 52, pp. 54731-54741, 2004.

[36] S. Cano-Crespo, J. Chillarón, A. Junza et al., "Cd98hc (slc3a2) sustains amino acid and nucleotide availability for cell cycle progression,” Scientific Reports, vol. 9, no. 1, p. 14065, 2019.

[37] C. Liu, X. Li, C. Li et al., "Slc3a2 is a novel endoplasmic reticulum stress-related signaling protein that regulates the unfolded protein response and apoptosis," PLoS One, vol. 13, no. 12, Article ID e0208993, 2018.

[38] X. Zhao, D. Wu, X. Ma, J. Wang, W. Hou, and W. Zhang, "Exosomes as drug carriers for cancer therapy and challenges regarding exosome uptake," Biomedicine \& Pharmacotherapy, vol. 128, Article ID 110237, 2020.

[39] S. Horibe, T. Tanahashi, S. Kawauchi, Y. Murakami, and Y. Rikitake, "Mechanism of recipient cell-dependent differences in exosome uptake," BMC Cancer, vol. 18, no. 1, p. 47, 2018.

[40] M. E. Vogel, G. Idelman, E. S. Konaniah, and S. D. Zucker, "Bilirubin prevents atherosclerotic lesion formation in lowdensity lipoprotein receptor-deficient mice by inhibiting endothelial vcam-1 and icam-1 signaling," Journal of American Heart Association, vol. 6, no. 4, 2017.

[41] R. Gotoh, J.-I. Suzuki, H. Kosuge et al., "E-selectin blockade decreases adventitial inflammation and attenuates intimal hyperplasia in rat carotid arteries after balloon injury," Arteriosclerosis, Thrombosis, and Vascular Biology, vol. 24, no. 11, pp. 2063-2068, 2004.

[42] X. Deng, X. Chu, P. Wang et al., "MicroRNA-29a-3p reduces TNF $\alpha$-induced endothelial dysfunction by targeting tumor necrosis factor receptor 1," Molecular Therapy-Nucleic Acids, vol. 18, pp. 903-915, 2019.

[43] R. Chenevard, D. Hürlimann, M. Béchir et al., "Selective cox-2 inhibition improves endothelial function in coronary artery disease," Circulation, vol. 107, no. 3, pp. 405-409, 2003. 